\title{
Imaging of Electric Fields at the GaN/Ni Interface Using Electron Beam Induced Current in a Scanning Transmission Electron Microscope
}

\author{
Zoey Warecki ${ }^{1}$, Vladimir Oleshko ${ }^{2}$, Andrew Armstrong ${ }^{3}$, Kimberly Collins ${ }^{4}$, A. Alec Talin ${ }^{4}$, John \\ Cumings ${ }^{1}$ \\ 1. Department of Materials Science and Engineering, University of Maryland, College Park, MD, USA \\ 2. Materials Science and Engineering Division, Material Measurement Laboratory, National Institute of \\ Standards and Technology, Gaithersburg, MD, USA \\ 3. Sandia National Laboratories, Albuquerque, NM, USA \\ 4. Sandia National Laboratories, Livermore, CA, USA
}

Gallium nitride is a promising wide bandgap material for demanding applications such as hightemperature and high-power electronics, as well as for space applications where its higher resistance to high fluxes of proton and electron radiation compared to Si represents an important advantage [1]. Electron irradiation of $\mathrm{GaN}$ is believed to create both $\mathrm{N}$ and $\mathrm{Ga}$ vacancies, as well as to induce threading dislocation glide. Typically, the creation or modification of structural defects in GaN are studied in a scanning electron microscope (SEM) retrofitted with cathodoluminescence (CL) or electron beam induced current (EBIC) tools, which identify locations of high defect concentration as dark regions due to increased (non-radiative) recombination rates. Unfortunately, the spatial resolution of these methods is limited due to the relatively large interaction volumes generated by primary electrons. While scanning transmission electron microscopy (STEM) enables the requisite spatial resolution necessary to understand the mechanisms of how defects are created and/or propagated under electron beam irradiation, it is still rarely combined with CL or EBIC, which can identify electronically active defects. Here we report the first STEM based EBIC characterization of Schottky diodes consisting of Ni contacts to free-standing hydride vapor phase epitaxy (HVPE) grown GaN with a threading dislocation density of $\sim 10^{5} / \mathrm{cm}^{2}$.

Our sample consists of a high purity single crystal n-type GaN substrate ( $275 \mu \mathrm{m}$ thick) grown by HVPE with a nickel Schottky contact as well as an indium ohmic contact. Specimen preparation for this sample was unconventional because we did not thin the sample as is typically done in TEM sample preparation. As can be seen from Figure 1a), the Schottky contact remains intact after mounting the sample to the Nanofactory holder. The Ni contact can be seen in both the optical micrograph as well as the energy dispersive $x$-ray spectroscopy (EDS) map in Figure 1b) and c). The bright field STEM image in the inset of Figure 2a) shows the outline of the GaN and $\mathrm{Au}$ wire but does not show any features, as expected due to the specimen thickness. However, the EBIC image only shows areas where charge carriers are measurably excited and separated, i.e. the $\mathrm{Ni} \mathrm{pad,} \mathrm{Ag} \mathrm{epoxy,} \mathrm{and} \mathrm{Au} \mathrm{wire.} \mathrm{Furthermore,} \mathrm{there} \mathrm{is} \mathrm{no}$ difference in the EBIC signal on or off the GaN, which indicates this is a true EBIC signal and not just a specimen current from e.g. secondary electrons.

In order to quantitatively interpret EBIC contrast, consideration of the beam/sample interaction is especially critical. During EBIC, the high energy electron beam excites electron-hole pairs in the material and, in the presence of an electric field, these charge carriers will separate and generate a current detectable with an ammeter [2]. In STEM, the interaction volume of the beam with a thick sample can be extremely large and thus decrease the resolution of the signal. We have begun Monte Carlo simulations to better understand our specific samples and plan to thin the samples in order to both restrict the beam-sample interaction as well as discern features in the sample with conventional STEM. Nevertheless, the average 
penetration depth of the beam into GaN can be approximated with Grün's empirical relationship: $R_{G}=$ $\frac{4.57 \cdot 10^{-6}}{\rho} E_{B}^{1.75}$, where $R_{G}$ is the penetration depth in $\mathrm{cm}, \rho$ is the density in $\mathrm{g} / \mathrm{cm}^{3}$, and $E_{B}$ is the energy of the beam in $\mathrm{keV}$ [3]. While this expression only estimates the interaction volume depth, it gives an order of magnitude approximation for the interaction volume radius. Using the expression above, $R_{G}$ for $\mathrm{GaN}$ equals 22 and $74 \mu \mathrm{m}$ for 100 and $200 \mathrm{kV}$ respectively. We thus tentatively assign the observed exponential decay of $4.4 \mu \mathrm{m}$ at $100 \mathrm{kV}$ and $14.8 \mu \mathrm{m}$ at $200 \mathrm{kV}$ to the interaction volume radius within the GaN.

This research was performed at the University of Maryland NanoCenter Advanced Imaging and Microscopy Laboratory. The data was taken on a JEOL 2100F TEM operating at $100 \mathrm{kV}$ and $200 \mathrm{kV}$ in STEM mode using a Nanofactory STM-TEM holder.

[1] S.J. Pearton, et al, ECS J. Solid State Sci. Technol., 5, Q35, (2016)

[2] H.J. Leamy, J. Appl. Phys., 53, (1982)

[3] A.E. Grün, Z Naturforsch, A J. Phys. Sci., 12, 89, (2016)

[4] The authors acknowledge funding from Nanostructures for Electrical Energy Storage (NEES), an Energy Frontier Research Center funded by the U.S. Department of Energy, Office of Science, the National Science Foundation Graduate Research Fellowship Program under Grant No. DGE 1322106, and support from NIST Grant No. 70NANB15H218. Sandia National Laboratories is a multi-mission laboratory managed and operated by Sandia Corporation, a wholly owned subsidiary of Lockheed Martin Corporation, for the U.S. Department of Energy's National Nuclear Security Administration under contract DE-AC0494AL85000. ZW thanks Dr. Wen-An Chiou and Dr. Sz-Chian Liou for their advice.
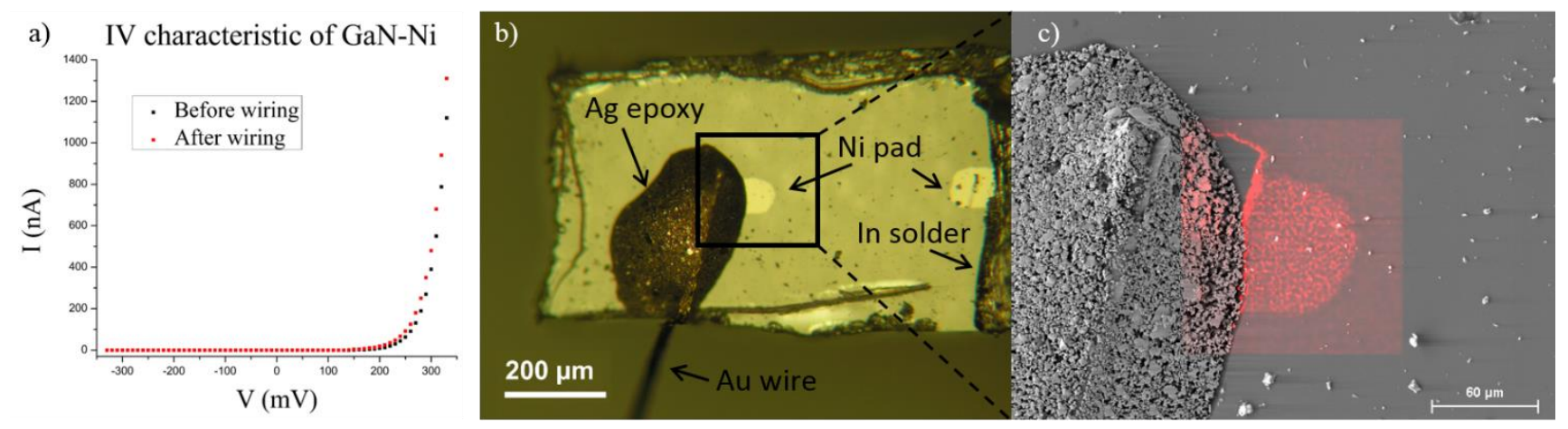

Figure 1. a) IV curve showing that no changes occurred to the GaN/Ni contact after sample preparation. b) Optical micrograph of sample after wiring, c) SEM image and overlaid EDS map showing Ni pad.
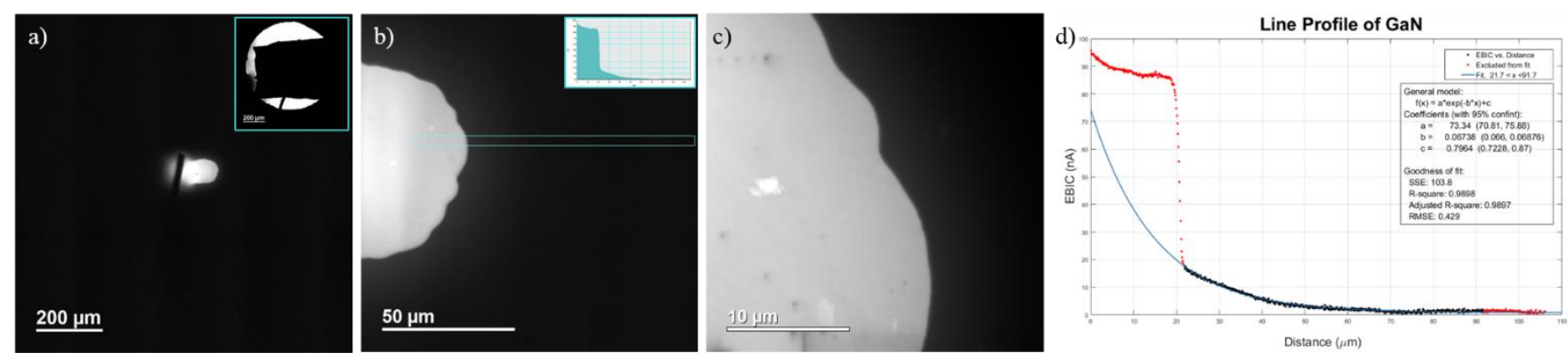

Figure 2. a) EBIC image at $200 \mathrm{kV}$ (inset BF STEM) b) EBIC at $200 \mathrm{kV}$ and c) EBIC at $100 \mathrm{kV}$ d) line profile fit illustrating how the interaction volume radius was estimated from data shown in the inset of c). 